\title{
Formative Assessment in China and Its Effects on EFL Learners' Learning Achievement: A Meta-Analysis from Policy Transfer Perspective
}

\author{
Qiuxian Chen", Huihui Li \\ School of Foreign Languages, Shanxi University, Taiyuan, Shanxi, China.
}

\begin{abstract}
How to cite this paper: Qiuxian Chen, Huihui Li. (2021). Formative Assessment in China and Its Effects on EFL Learners' Learning Achievement: A Meta-Analysis from Policy Transfer Perspective. The Educational Review, USA, 5(9), 355-366. DOI: 10.26855/er.2021.09.005
\end{abstract}

Received: August 9, 2021

Accepted: August 31, 2021

Published: September 27, 2021

Corresponding author: Qiuxian Chen, School of Foreign Languages, Shanxi University, Taiyuan, Shanxi, China.

Email: chenqx@sxu.edu.cn

\begin{abstract}
Black and Wiliam's seminal review elicited an impressive effect size ( $d=0.4-0.7)$ for the efficacy of formative assessment, which in turn became the very foundation for the prevalence of formative assessment in theory, policy and practice around the globe. This effect size, however, has been questioned by meta-analyses from America, French and Spanish sources. With an eye on the policy transfer across borders and cultures, this paper explored, via a meta-analysis, how, if yes, the efficacy of formative assessment has been transferred in English as a Foreign Language (EFL) education area in the Chinese context. Analysis of 33 effect sizes from eligible 27 studies revealed a medium-sized positive effect of formative assessment on Chinese students' EFL learning (0.46). Subsequent moderator analysis showed that formative assessment seemed to work better on students' vocabulary learning (0.84) and spoken language achievement (0.65) than reading, writing and listening, and on small-sized groups (0.69) than larger ones (-0.12). Implications for pedagogy and future policy-making were derived therefrom.
\end{abstract}

\section{Keywords}

Formative Assessment, China, EFL, For-Learning Function, Policy Transfer

\section{Introduction}

Black and Wiliam (1998a; 1998b) concluded out of a systematic review of 250 studies published in English journals that formative assessment could lead to substantial learning gains (effect size of 0.4-0.7), and was hence one of "the most powerful tools" to raise educational standards. This conclusion has brought formative assessment to the fore, and is seen as responsible, in a large part, for the globe-wide assessment-related innovations in the past two decades (Berry \& Admson, 2011; Brown et al., 2019). This conclusion, however, is not without critiques. For instance, Hattie and Timperley (2007) and Shute (2007), while confirming the potential power of formative feedback on learning achievement, have highlighted the complexities involved over the process, and noted that it need to satisfy necessary conditions and have proper mechanisms before its power could be realised in practice. Dunn and Mulvenon (2009), on the other hand, critiqued the methodologies and findings of the eight major studies that Black and Wiliam (1998a) relied heavily on in their review and nine empirical studies published over the following decade, and commented that the scientific evidence of the learning potentials of formative assessment was "limited", and need to be further supported. Likewise, Bennett (2011, p. 5) argued that Black and Wiliam's (1998a) efficacy argument of formative assessment was far from conclusive, since the scientific sources it derived from, particularly those quantitative studies, were "untraceable, flawed, dated, or unpublished". All these critiques give rise to the concern to what degree, if yes, the adoption and adaptation of formative assessment in multiple educational settings could lead to a positive transfer of the effect size, and more im- 
portantly, its learning promises.

The transfer of formative assessment and its learning potentials across contexts have been the focus of a few investigations. The analysis of 105 studies from French speaking settings (including France, Belgium, Canada, and Switzerland) (Allal \& Lopez, 2005) revealed that only four studies presented conservative and inconsistent findings on the effectiveness of formative assessment. The first study showcased positive effects in the first semester, yet failed to maintain it later on; the second study witnessed a substantial improvement of student attitudes toward assessment but a relatively small effect on spelling scores; the third, a quasi-experimental study carried out in Québec, reported a positive effect size of 0.56 for achievement in subjects like French and mathematics, but little information on the methodologies were available to prove its validity. The last one failed to rule out influences from diagnostic technology. In brief, empirical evidence of formative assessment's positive effects on learning was rather limited from French sources.

In America context, Kinston and Nash's (2011) meta-analysis of 13 eligible literature on formative assessment's impact on the K-12 students revealed a mean effect size of $(d=0.20)$, and an estimated effect of $0.32,0.17$, and 0.09 for English language arts, mathematics or science subjects respectively. Later, Klute et al. (2017) derived 30 effect sizes from 19 eligible studies on elementary school students and revealed a mean positive effect of formative assessment $(d=$ $0.26)$, which varied by subject areas: mathematics $(d=0.36)$ and reading $(d=0.22)$. Lee et al.'s $(2020)$ review of a larger number of and more recent literature (33) yielded a slightly higher mean effect size $(d=0.29)$ of formative assessment on the American K-12 students' learning and individual effect of 0.34 in mathematics, 0.33 in literacy, and 0.29 in arts. All these were much lower than the 0.40 to 0.70 value that was identified in Black and Wiliam (1998b). Their fellow American, Graham et al. (2015), however, presented a different picture. He analysed 27 studies on the impact of formative feedback on American 1 to 8 graders' writing performance and computed an overall effect size of 0.61 , which was comparable to that Black and Wiliam (1998a) generated from the English sources. These findings from American context point to the possibility that the learning potentials of formative assessment might as well be discipline or subject dependent.

Indeed, Flórez and Sammons' (2013) qualitative analysis of 33 highly relevant literature of English and Spanish sources from 1998 to 2010 revealed that while the positive effects of formative assessment on student learning and achievement were well perceived by participants, solid statistical evidence from this setting was confined to a few experimental studies only. For further research, he emphasised the significance of rooting within specific content domains.

This study was thus designed to investigate the effects of formative assessment on students' learning in the English as a Foreign Language (EFL) area of Chinese context with literature of English and Chinese sources. Given the sociocultural, historical and system discrepancies of the Chinese context from the Anglophone contexts that the formative assessment principles were originated and developed, this meta-analysis hopes to add to nuanced understanding about the complexities involved in the policy transfer of formative assessment across borders and cultures.

\section{Background}

Starting from early 2000s on, the Chinese Ministry of Education (CMoE) has endeavored to introduce formative assessment to its education of various disciplines. The EFL area, for example, has seen the issuing of national syllabi such as National English Curriculum Standards for Basic Education (CMoE, 2001), National English Curriculum Standards for Senior High School (CMoE, 2003), and College English Curriculum Requirement (CMoE, 2007) for EFL education of basic (year 1-9), secondary (year 10-12), and tertiary level respectively. All these documents advocated formative assessment for the purpose of improved teaching and learning, as well as better implemented curricula.

Formative assessment, while gaining a legitimate way into the EFL area of China via these documents, was confronted with a deeply-entrenched testing dominated regime. Indeed, assessment in this area was virtually equivalent to testing, or standardised testing more exactly (Jin \& Sun, 2020). Multiple-choice questions abound in EFL tests from primary to tertiary and beyond. Particularly, English took up a handsome share (15\%-20\%) of the Senior High Entrance Examination (referred to as zhōngkăo), the National College Entrance Examination (referred to as gāokăo), and the National Graduate Entrance Examination (kăoyán), the gate-keeping examinations to senior high, university and graduate schools. As students' performance in these tests contributed to, if not determined, their chances of success to next level of education, these examinations tended to control the EFL teaching and learning as a virtual curriculum (Chen et al., 2014; Qi, 2007). More importantly, a mindset which highlighted the utilitarian values of education, the key role of examination in education and the one-off result of assessment (Han \& Yang, 2001) is widely accepted in the context (Cheng \& Curtis, 2009). In other words, a testing culture prevails in the EFL classrooms and, more generally, in the wider context of Chinese society.

The adoption of formative assessment against this backdrop, therefore, has led to "two competing assessment discourses”, that is, the existing summative and the emergent formative, in the EFL education area of China (Liu \& Xu, 
2017, p. 22). While the former aimed for accountability and selection purposes, the latter was striving for learning enhancement. The resultant tensions had put stake-holders such as teachers and students in a dilemmatic circumstances (Rea-Dickins, 2004), and made it very hard for the formative assessment initiatives to establish and effect the change(s) as intended in the initiatives (Carless \& Lam, 2014).

\section{The inroads of formative assessment in the EFL area of China}

The hard-going inroads of formative assessment into the Chinese EFL context are recaptured in a few literature reviews. Huang and Luo's (2014) synthesis of the published articles, monographs in Chinese journals and educational newspapers and unpublished $\mathrm{PhD}$ dissertations from 2001 to 2012 concluded that formative assessment in China was faced up with multiple contextual challenges, logistic constraints as well as the obsolescence of pedagogy and assessment literacy and other predicaments. Regardless of the arising policy and scholarly passion, they found formative assessment in China was still in "a budding stage” (Huang \& Luo, 2014, p. 24), and was "inadequately researched, poorly understood and improperly implemented" (Huang \& Luo, 2014, p. 19). They saw in their review little attending to the effects of formative assessment in the Chinese context. In contrast, their more recent review of 97 publications from the Chinese high-ranking journals shed a different light: the effects of formative assessment seemed optimistic and promising and were evidenced with nearly all experimental studies included in the review (Huang, Luo, \& Lin, 2019). Pitifully, they failed to go deep in this regards. Yuan and Shu's (2017) review, which included 158 articles published in 18 key journals of the EFL field from 2002 to 2016, touched upon the efficacy of formative assessment in China as well. They did not go deep either except a cautionary note that the largely positive findings of the 21 empirical studies need to be further verified.

Likewise, the efficacy of formative assessment in China was presented in Jin and Sun's (2020) review of articles published in 5 SSCI and 9 CSSCI journals during the period of 2007-2018 in a brief and reserved way as a theme: being positive in oral and writing classes yet not quite salient with College English learning outcomes. Min et al. (2020) commented with their review of most recent English language assessment literature of Chinese source that the value of formative assessment was yet to be recognized and how to maximize learning gains through assessment was yet to be explored in the Chinese contexts. These reviews seem to reach a consensus that regardless of the policy and scholarly inspirations for formative assessment and its for-learning promises, the inroads of formative assessment into the Chinese context have been difficult and complicated, and so far, the efficacy of formative assessment in the EFL area of China remains an agenda underexplored. This study hence endeavors to fill in the gap with a focused meta-analytic investigation into the association of formative assessment interventions and its learning potential in this particular context.

As such, the present analysis synthesized the results of available and relevant studies to address two research questions:

1) How has formative assessment impacted on Chinese students' English learning achievement?

2) How has the efficacy of formative assessment on Chinese students' English learning been moderated?

\section{Methodology}

\subsection{A meta-analysis approach}

Meta-analysis is a statistical tool often used to (1) synthesis the quantitative results of a set of empirical studies conducted to answer the same research question(s) to obtain a mean effect size as "a proxy of the population effect" and (2) identify the moderating factors across studies so as to explain the variations of the effects and/or relationships therein (Li \& Wang, 2018, p. 133). It suits well this study's need for an overall evaluation of the effectiveness of formative assessment in the Chinese EFL context and a tentative investigation into the possible influences. Also, it is the method that many of the reviews of other sources (Allal \& Lopez, 2005; Kinston \& Nash, 2011; Klute et al., 2017) used to argue for or against the efficacy of formative assessment. This study chose meta-analysis so that its results can be compared with that of other reviews.

To address the research problem, this meta-analysis has amalgamated the findings of available studies that focus on the impact of formative assessment on Chinese EFL students' achievement, and has gone through a set of rigorous procedures: searching for and selecting/excluding studies in the synthesis, coding, analysing, and writing up (Li \& Wang, 2018), which is detailed below.

\subsection{Literature search and criteria for inclusion}

The database search, as a common search strategy in meta-analysis (Li \& Wang, 2018), was adopted to locate literature to be included. Specifically, we cross-searched with keywords "formative assessment/formative evalua- 
tion/assessment for learning”, "English” and "China” on ERIC, EBSCO, two large scale databases of English source. Then, we cross-searched with key words "Formative evaluation/formative evaluation/learning promotion evaluation" and "English" on CNKI, the most influential Chinese database in China. The time span was set between "2000" and "present" because formative assessment was introduced to China after 2000, and few research was available before the time. It needed noting that we limited the search on CNKI to CSSCI, which was the up-to standards journal list of Chinese sources acknowledged in the academic field of China, to ensure the included studies were up to standards as well.

The database retrieval from English and Chinese sources firstly identified 2,248 articles. Then, title and abstract of these articles were scanned through, which narrowed the dataset down to 201 relevant articles. The methodology and finding sections of these studies were then read closely referring to four criteria: 1) description of the intervention of formative assessment/evaluation or assessment for learning, with a focus on its effects on student learning achievements particularly; 2) empirical studies of quantitative nature; 3) control group or comparison group design; 4) inclusion of appropriate statistics for the calculation of effect sizes. The process, as shown in the flow chart (Figure 1), identified 27 eligible articles to be included in the meta-analysis.

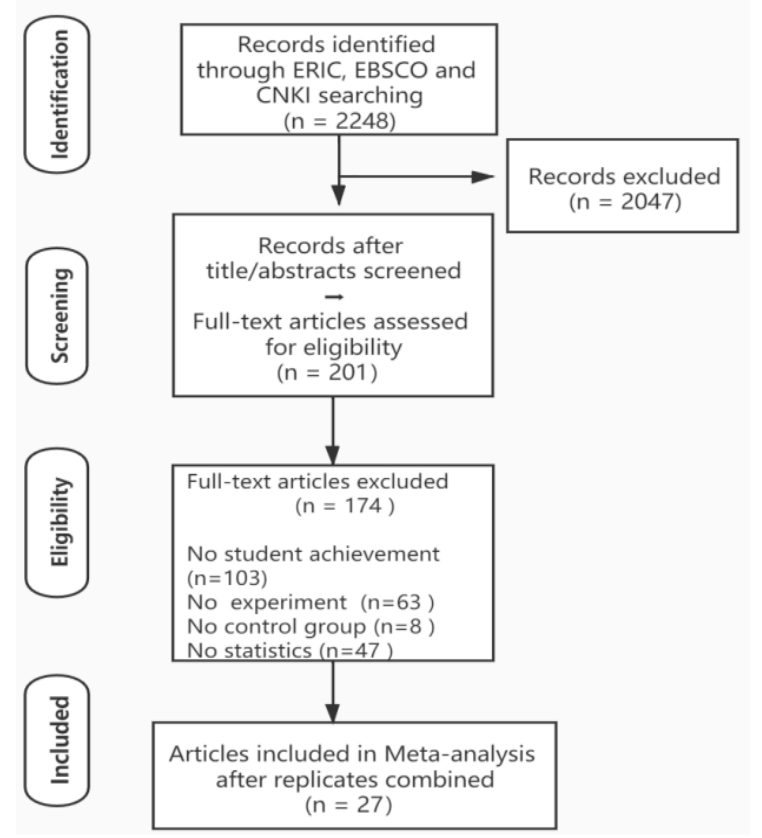

Figure 1. PRISMA Flow Chart (adapted from Moher et al., 2009).

\subsection{Coding scheme}

The 27 identified articles were read in full and coded. The coding firstly revealed treatment types of various emphases (see Table 1).

Table 1. Treatment type emphases

\begin{tabular}{cc}
\hline Treatment & Frequencies \\
\hline Engaging Student in assessment & 11 \\
Use of feedback & 11 \\
Portfolio & 8 \\
Computer-based formative system & 7 \\
Curriculum-embedded assessment & 2 \\
Others (Conferencing, Logs, journals or Observation of assessment activities) & 10
\end{tabular}

As can be seen from the table, the 27 studies have employed formative strategies such as student engagement in assessment, feedback, portfolio, computer-based formative system, curriculum-embedded assessment etc. The use of these strategies indicated that these studies did involve formative intervention. It needed noting, however, that individual studies adopted most often more than one treatment type, and the findings of the 27 studies were presumably the 
combined results of treatments employed.

The 27 articles were also coded on dimensions such as subject area, educational level, sample size, and experiment duration. In the coding process, four studies emerged, which reported data of different non-overlapping demographics, such as multiple subjects (Wang, 2006; Chen, 2007), different classrooms (Tang \& Zhang, 2007) or academic years (Wang, 2011). As Borenstein et al. (2009) viewed groups as superior to the calculation of an average effect size for the study as a whole because groups differed across potential moderator variables. These subgroups (10 altogether) were therefore treated as statistically independent in this study. This meta-analysis hence ended up with 33 items in total (see Table 2).

Table 2. Studies included in the meta-analysis \& their demographics as coded

\begin{tabular}{|c|c|c|c|c|c|}
\hline \multicolumn{2}{|c|}{ Author and year } & $\begin{array}{c}\text { Subject area } \\
\text { Comprehensive English }\end{array}$ & $\begin{array}{c}\text { Sample size } \\
58\end{array}$ & $\begin{array}{c}\text { Educational level } \\
\text { Senior }\end{array}$ & $\begin{array}{c}\text { Duration } \\
3 \text { semesters }\end{array}$ \\
\hline \multirow{3}{*}{ Wang (2006) } & Subject 1 & Listening & & & \\
\hline & Subject 2 & Reading & 301 & Tertiary & 2 years \\
\hline & Subject 3 & Writing & & & \\
\hline Wang \& Ma (2006) & & Reading & 301 & Tertiary & 1 semester \\
\hline \multirow{2}{*}{ Chen (2007) } & Subject 1 & Comprehensive English & \multirow{2}{*}{104} & \multirow{2}{*}{ Tertiary } & \multirow{2}{*}{1 semester } \\
\hline & Subject 2 & Spoken English & & & \\
\hline \multirow{2}{*}{ Tang \& Zhang (2007) } & Group 1 & Writing & \multirow{2}{*}{559} & \multirow{2}{*}{ Tertiary } & \multirow{2}{*}{12 weeks } \\
\hline & Group 2 & Writing & & & \\
\hline Mo (2007) & & Writing & 49 & Tertiary & 7 weeks \\
\hline Zhang (2008) & & Comprehensive English & 117 & Senior & 1 semester \\
\hline Cao et al. (2008) & & Comprehensive English & 1000 & Tertiary & 15 weeks \\
\hline Xiao \& Cao (2009) & & Listening & 60 & Tertiary & 1 year \\
\hline Zhang (2010) & & Comprehensive English & 120 & Tertiary & 1 year \\
\hline \multirow[t]{2}{*}{ Man (2011) } & & Comprehensive English & 77 & Senior & 1 semester \\
\hline & Group 1 & Writing & & & \\
\hline \multirow[t]{2}{*}{ Wang (2011) } & Group 2 & Writing & 900 & Tertiary & 8 weeks \\
\hline & Group 3 & Writing & & & \\
\hline Zhou (2011) & & Comprehensive English & 43 & Tertiary & 1 year \\
\hline Jiang (2011) & & Writing & 92 & Tertiary & 1 semester \\
\hline Feng \& Gao (2012) & & Writing & 63 & Tertiary & 1 year \\
\hline Yu (2013) & & Listening & 195 & Tertiary & 1 year \\
\hline Zheng \& Li (2014) & & Spoken English & 56 & Tertiary & 16 weeks \\
\hline Li (2014) & & Comprehensive English & 48 & Tertiary & 2 years \\
\hline Tang (2014) & & Writing & 303 & Senior & 2 semesters \\
\hline Huang (2015) & & Writing & 149 & Tertiary & 15 months \\
\hline Zhang \& Wei (2015) & & Spoken English & 76 & Senior & 1 year \\
\hline Feng (2015) & & Vocabulary & 66 & Senior & Not reported \\
\hline Bi (2017a) & & Vocabulary & 70 & Tertiary & 6 weeks \\
\hline Bi (2017b) & & Listening & 66 & Tertiary & 12 weeks \\
\hline Zhang (2017) & & Writing & 59 & Tertiary & 1 semester \\
\hline Chen \& Li (2017) & & Writing & 57 & Tertiary & 1 year \\
\hline Zhang \& John (2020) & & Writing & 198 & Tertiary & 15 weeks \\
\hline
\end{tabular}

As Table 2 shows, six of the 33 groups involved students of senior level and 27 (81.8\%) of tertiary level. That is, eligible studies on formative assessment at the elementary level were rare. 14 out of 33 groups were based on English writing, eight on comprehensive English, four on English listening, three on spoken English, and two on English reading and vocabulary, covering most of the subareas of the English subject. Duration ranged from six weeks to two years, 
with those lasting for one semester (9) or one year (7) being the most frequent. Sample sizes varied too from 43 to 1,000 student participants. These variations were likely to make this study more robust. These data were then put into the Comprehensive Meta-Analysis (CMA) ${ }^{1}$ software (3.0) as covariates to investigate the issue in focus.

\subsection{Analysis}

The data went through a four-stage analysis. Firstly, calculate the effect sizes. Based on Cohen's $d$ Effect Size (ES) calculation (Cohen, 1988), which is most frequently used in meta-analysis. We ran CMA to compute unbiased $d$ (namely, standardized differences in means). Secondly, test the heterogeneity. The test was taken to check if the percentage of variation across studies was due to heterogeneity rather than chance (Borenstein et al., 2009). We assessed the heterogeneity between these effect sizes and determine the relationships among study variations using the $Q$ value and I-squared index $\left(I^{2}\right)$ (Borenstein et al., 2009). Thirdly, analyse publication bias. We assessed publication bias with visual inspection of a funnel plot, which was plotted with effect size on the x-axis and standard errors of the effect size on the y-axis (Borenstein et al., 2009). Lastly, meta regression and moderator analysis. Given the significant heterogeneity between studies, we used a meta-regression analysis to identify possible moderating variables and the degree of the moderation to explain potential study heterogeneity (Borenstein et al., 2009). The four-stage process generated results regarding the effectiveness of formative assessment in the English education area of China as below.

\section{Results}

\subsection{Effect sizes calculation}

An effect size, that is, the differences between means, was calculated with CMA for each of the 33 studies to quantify the relationships between the independent variable (use of formative assessment strategies) and dependent variable (student achievement). Consequently, 33 effect sizes and a forest plot of the studies were resulted (Figure 2).

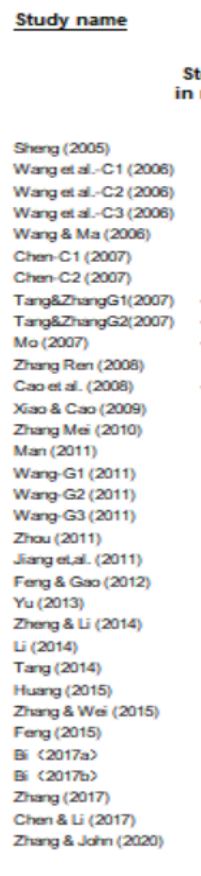

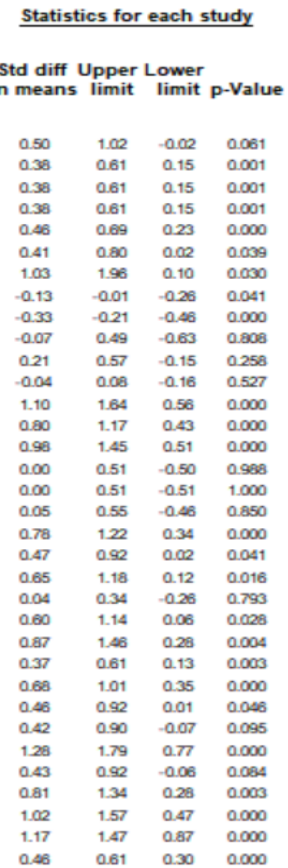

Std diff in means and $95 \% \mathrm{Cl}$

Figure 2. Effect sizes calculated and forest plot.

As Figure 2 showed, the weighted mean effect size across studies was $d=0.46$ with a $95 \%$ confidence interval of 0.30 to 0.61 . In the forest plot, the effect sizes, which varied substantially from one study to another, were depicted as point estimates bounded by its confidence interval. And all but four effect sizes, that is 29, were positive. The 29 positive effect sizes out of the 33 indicated that formative assessment interventions most likely have affected Chinese students’

${ }^{1}$ Comprehensive Meta-Analysis, as the name suggests, is an indispensable tool for conducting a meta-analysis, it is particularly convenient for computing the effect size automatically with summary data such as sample size, means and standard deviations or p-value or t-value, and assessing the heterogeneity of these effect sizes and the impact of moderator variables (Borenstein et al., 2009). 
EFL learning achievements positively.

\subsection{Heterogeneity test}

This result was then put to a heterogeneity test to verify the variation across studies was due to heterogeneity rather than chance (Borenstein et al., 2009). A running of CMA presented a result as showcased in Table 3.

Table 3. The Heterogeneity Test

\begin{tabular}{ccc}
\hline \multicolumn{3}{c}{ Heterogeneity } \\
\hline $\mathrm{Q}$ & $\mathrm{P}$ & $\mathrm{I}^{2}$ \\
259.90 & 0.000 & 87.69 \\
\hline
\end{tabular}

The $Q$ statistic of 259.90 indicated that the variances among effect sizes were far greater than expected by chance $(p$ $<0.001$ ). The $I^{2}$ value $^{2}$ of 87.69 , which was above the $75 \%$ benchmark, similarly suggested $87.69 \%$ of the variation of effect sizes could not be explained by the sampling distribution of study participants. That means a significant heterogeneity existed across the studies. Put differently, the 33 studies were largely heterogeneous, their effect sizes could be treated as samples from different but related populations of experiments. This result supported the use of a random effect approach to the meta-analysis.

\subsection{Publication bias analysis}

Following the meta-analysis procedures (Borenstein et al., 2009), publication bias of these studies was firstly assessed using the funnel plot for asymmetry (Figure 3).

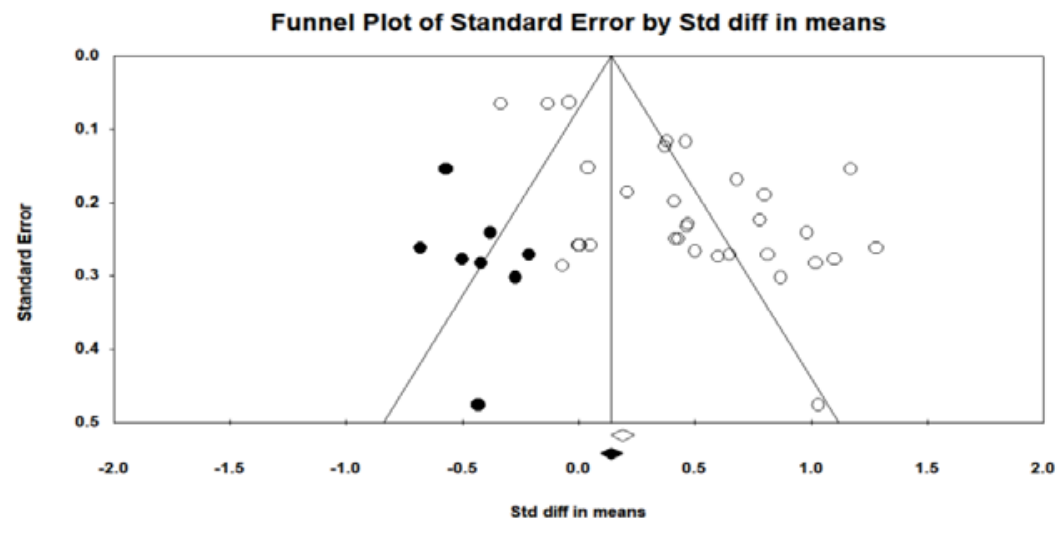

Figure 3. Formative assessment and students' achievement-funnel plot.

Visual inspection of the funnel plot revealed a potential publication bias because the observed studies, shown as open circles, were distributed quite asymmetrically around the mean $(d=0.16)$. This necessitated the running of an Egger's test, which was usually used to declare the statistical significance of publication bias (Borenstein et al., 2009). Egger's test revealed a statistically significant p value of 0.0000 (Table 4), which confirmed the existence of publication bias.

Table 4. Egger's test

\begin{tabular}{ccccc}
\hline & $\begin{array}{c}\text { 95\% Confidence Interval } \\
\text { mean }\end{array}$ & upper & Standard Error & P \\
\hline 2.46 & 3.90 & 5.33 & 0.70 & 0.000 \\
\hline
\end{tabular}

The trim-and-fill and Rosenthal's fail-safe $N$ were further employed to assess whether the bias would influence the results of meta-analysis. The trim-and-fill adjustment showed that eight imputed studies (shown as filled circle in

\footnotetext{
${ }^{2}$ The value of $I^{2}$ ranges of $25 \%, 50 \%$, and $75 \%$ have been suggested as benchmarks of low, moderate, and high heterogeneity. That is, if $I^{2}<50 \%$, all the studies are homogeneous, then a fixed effect size model could be used for combined analysis; $I^{2} \geq 50 \%$, on the other hand, means a significant heterogeneity among the studies, which indicates some variabilities among the individual effect sizes and a further need to focus on the causes. In such cases, a random-effects model is applicable (Borenstein et al., 2009).
} 
Figure 3) were needed on the left side to make the funnel plot symmetrical (Duval \& Tweedie, 2000). That means the publication bias needed eight more studies to be leveled off. The results from Rosenthal's fail-safe $N$, on the other hand, indicated that a non-significant $p$ value needed 719 additional studies to be produced (Table 5).

Table 5. Rosenthal's fail-safe N

\begin{tabular}{ccc}
\hline & Rosenthal's fail-safe $\boldsymbol{N}$ & \\
\hline Observed studies & Missing studies & $\mathrm{P}$ \\
33 & 1,251 & 0.000 \\
\hline
\end{tabular}

The number of 1,251, however, far exceeded Rosenthal's criterion of $5 k+10=175$ (with k referring to the effect size number, $k=33$ in this case), indicating that publication bias has little influence on the results of meta-analysis in this study.

With heterogeneity test and publication bias both verified, it is now safe to say that formative assessment was largely effective in the English education area of China with a mean effect size of $d=0.46$.

\subsection{Moderator and meta-regression analysis}

The four covariates (subject area, educational level, sample size and duration) revealed in the coding process were regressed to account for the excess variability, that is, to test whether they could explain some of the between-study variation in the (uncorrected) correlations (Borenstein et al., 2009). A meta-regression analysis via random-effect model identified the possible moderators to the effectiveness of formative assessment in the English education area of China as below (Table 6).

Table 6. R-squared for each model

\begin{tabular}{ccccc}
\hline \multirow{2}{*}{ Model } & \multicolumn{4}{c}{ R-squared for each model } \\
\cline { 2 - 5 } & Total variance & Not explained by model & Explained by model & R-squared \\
\hline duration & 0.1257 & 0.1257 & 0.0000 & 0.00 \\
educational level & 0.1257 & 0.1254 & 0.0003 & 0.00 \\
subject area & 0.1257 & 0.1121 & 0.0136 & 0.11 \\
sample size & 0.1257 & 0.0343 & 0.0914 & 0.73 \\
\hline
\end{tabular}

According to Cohen's (1988) criterion of correlation coefficient $R^{2}$, the duration and education level variables were basically not correlated (0.00) with the results regarding efficacy of formative assessment in the English area of China; subject area was weakly correlated (0.11); yet, sample size was strongly correlated (0.73). That is to say, the educational level and duration did not account for a significant amount of the variability of effect sizes. Or say, formative assessment could be effective to the Chinese students English learning no matter how long or how short it was and no matter which educational level the subjects were from. Two correlated variables (subject and sample size) were adopted as significant moderators to further explore the relationship between study features and students learning achievement. The results of analysis were showcased respectively in Tables 7 and 8.

Table 7. Moderator analysis for subject area

\begin{tabular}{|c|c|c|c|c|c|c|}
\hline \multicolumn{7}{|c|}{ Effect Size and 95\% Confidence Interval } \\
\hline Subject type & $\mathrm{n}$ & Lower & Mean & Upper & Standard Error & Variance \\
\hline Writing & 14 & 0.10 & 0.23 & 0.36 & 0.13 & 0.02 \\
\hline Comprehensive English & 8 & -0.33 & 0.44 & 1.21 & 0.70 & 0.30 \\
\hline Listening & 4 & -0.02 & 0.46 & 0.93 & 0.24 & 0.60 \\
\hline Spoken English & 3 & 0.03 & 0.65 & 1.27 & 0.32 & 0.10 \\
\hline Vocabulary & 2 & 0.13 & 0.84 & 1.55 & 0.36 & 0.13 \\
\hline Reading & 2 & -0.48 & 0.44 & 1.36 & 0.46 & 0.21 \\
\hline Total & 33 & 0.28 & 0.49 & 0.69 & 0.10 & 0.01 \\
\hline
\end{tabular}


Table 8. Moderator analysis for sample size

\begin{tabular}{|c|c|c|c|c|c|c|}
\hline \multicolumn{7}{|c|}{ Effect Size and 95\% Confidence Interval } \\
\hline Sample size & $\mathrm{n}$ & Lower & Mean & Upper & Standard Error & Variance \\
\hline$<100$ & 15 & 0.52 & 0.69 & 0.85 & 0.08 & 0.00 \\
\hline $100-500$ & 12 & 0.34 & 0.48 & 0.62 & 0.07 & 0.01 \\
\hline $500-1,000$ & 6 & -0.31 & -0.12 & 0.07 & 0.10 & 0.01 \\
\hline Total & 33 & -0.08 & 0.35 & 0.78 & 0.22 & 0.05 \\
\hline
\end{tabular}

As Table 7 shows, formative assessment interventions seemed to work on all the involved subject areas, only with varied degrees. Specifically, formative assessment worked the best on Chinese students' English vocabulary learning $(d$ $=0.84)$ and spoken language achievement (0.65), moderately on comprehensive English (0.44) and reading (0.44), and listening (0.46), and not so well on English writing subject (0.23).

Table 8, on the other hand, revealed that formative assessment interventions seemed to work progressively better on smaller samples, with sample size of less than 100 being the best (0.69), followed by sample size of 100-500 (0.48), and then sample size of $500-1,000(-0.12)$. It needs noting that -0.12 value means, when sample size goes between 500-1,000, formative assessment would affect student learning negatively rather than positively.

\section{Discussion}

The above results were synthesised to address the two research questions as below:

\subsection{Discussion related to the first research question}

The question "How has formative assessment impacted on Chinese students' English learning achievement?" aimed for the overall evaluation of the efficacy of formative assessment in the Chinese EFL learning context. As presented above in the result section, the calculation of 33 effect sizes from 27 studies has revealed a mean effect size of 0.46. This value falls between the classic 0.4-0.7 range that Black and Wiliam (1998a) reported in their seminal review, close to the low end though. With this result being verified by heterogeneity test and publication bias analysis, it tends to address the first research question in a positive way. That is, formative assessment did and could impact on the Chinese students' English learning positively.

Considering the testing-dominance within the EFL discipline (Jin \& Sun, 2020; Cheng \& Curtis, 2009) and the examination tradition (Han \& Yang, 2001; Kennedy, 2016) in the Chinese context, this result is rather encouraging, and indicates, in some sense, that two decades' policy efforts seems to have yielded some good produces in the Chinese context. This result is rather promising as well, given the formative assessment in China is still in a "budding stage" (Huang \& Luo, 2014, p. 24) and the Chinese government is keen to trigger off a changing climate to the educational assessment, with the new round of assessment reform (CPC, 2020) under way. This, however, is not a reason good enough for optimism yet, because cautions against the reliability and validity of these formative assessment-related studies are many (Huang \& Luo, 2014; Yuan \& Shu, 2017; Huang, Luo, \& Lin, 2019). Moreover, the multiple challenges and contextual constraints that formative assessment had to face up within the context, such as high-stakes testing, limited assessment literacy, obsolescent pedagogy, and particularly, the deep-rooted ideologies and mindsets (Carless \& Lam, 2014; Chen, 2016; Liu \& Xu, 2017; Poole, 2016) are not to be resolved within a short period of time. That means a lot still need to be done before the learning promises of formative assessment are realised in the Chinese context. A good news is the Chinese government (2020), well aware of the issues and keen to remove the barriers to its education quality, has lately issued a policy_ “The overall plan for deepening the reform of education evaluation in the new era”. With this new move, a system wise innovation was launched to effect ground-breaking changes on its educational assessment in terms of mechanism, orientation, and more importantly, assessment culture. Hopefully, the overall assessment environment in the Chinese context will be able to afford rather than constrain the uptake of formative assessment.

\subsection{Discussion related to the second research question}

The second research question "How has the efficacy of formative assessment on Chinese students' English learning been moderated?" focused on the possible influences on the efficacy of formative assessment in the selected area. Moderator analysis in above section showed that the results were not influenced by variables such as duration and educational level, yet correlated slightly with subject area (0.11) and significantly with sample size (0.73).

Further analysis revealed that formative assessment was effective to all the subject areas in varied degrees. Of the 
many subject areas, English vocabulary learning (0.84) and speaking (0.65) appear to be more sensitive to formative interventions than comprehensive English (0.44), reading (0.44), listening (0.46), and English writing (0.23). This finding agrees with Yuan and Shu's (2017) observation that formative assessment largely has a positive impact on Chinese students' EFL learning, yet consistent only in part with Jin and Sun's (2020) finding that formative assessment is positive with Chinese EFL oral and writing class, but not quite obvious with College English (that is equivalent to comprehensive English in this study) learning outcomes. While the inclusion of different sources with different criteria might account for the difference, the quality of the experiment and teachers' different implementation of formative assessment could be another possible reason for the different effects on different subjects (Yin, 2005). Kingston and Nash (2011) and Wiliam (2018), on the other hand, have unanimously presumed that the different effects may lie in the nature of content areas, which means, different subjects may have varied sensitivity to the effects of formative assessment interventions. This we agree, and observe from these findings that formative assessment seems to work better with areas which are easy to operate, such as vocabulary, than writing, which is widely acknowledged as the hardest of the four English language skills (Darwish \& Sadeqi, 2016). This observation, however, contradicts with the 0.61 value of Graham et al. (2015) with American students of primary level. The fact that none of the studies included in this paper are of primary level and writing proficiency improvement at the beginning stage is more visible and detectable than that of higher levels could explain this discrepancy in part. Also notable is that, in contrast to American students' using English as a native tongue, the Chinese students learn English as a foreign language. Since it is well-acknowledged that different English learning contexts such as inner circle, outer circle and expanding circle might lead to totally different English learning approach and results (Kachru, 1996), we assume the different language learning contexts are a likely explanation for the different efficacy in American and Chinese students' English writing as well. The correlation of this difference with the low/high efficacy of formative assessment needs, however, further and more focused investigation to verify.

The exploratory analysis also found that the efficacy of formative assessment tends to decrease with the sample size. Smaller sample size is more likely to achieve better formative assessment efficacy; large sample size (500-1,000, for instance), on the other hand, might induce negative (-0.12) rather than positive effect on students' English learning. This finding confirms the challenges that large class size poses to formative assessment practice (Liu \& Xu, 2017; Xu \& Harfitt, 2018) and in a sense reveal a pre-requistie for effective formative assessment: the class size needs to be small. Otherwise, all the efforts could be in vain, or worse still, lead to reverse effects.

\section{Conclusion}

Results as such point to the conclusion that despite all the challenges and contextual constraints, the for-learning function of formative assessment has been transferred to China, in some degree, and in the English education area at least. In other words, the learning potentials of formative assessment is transferable across contexts and cultures. This encouraging note could in turn boost deeper and wider application of formative assessment in the Chinese and other contexts.

The results imply a lot for classroom pedagogy and policy which are keen to bring the for-learning promises of formative assessment into reality. Firstly, formative assessment may not work well with big class; small class could be necessary to optimize feedback and classroom interactions, and hence better learning. Secondly, formative assessment practice in different subject areas may need to be tailored according to their different sensitivity degrees. Thirdly, assessment policy and its orientation might lead to totally different responses and learning outcomes. The No Child Left Behind Policy (2001), which highlighted the accountability purpose of assessment could be responsible for the low efficacy of formative assessment in the American context. The proactive policy and active practice in the Chinese context, on the other hand, are closely linked to the encouraging results as revealed in this study.

This findings also points to a few areas for further researches. For example, subjects other than EFL in China could be a good area for future exploration so as to present a fuller scenario about the efficacy of formative assessment in the context. For another example, China-based studies could put more attention to students of lower educational levels such as primary and junior high, so as to provide deeper and aggregated insights into the efficacy of formative assessment in the context and beyond. Also, more moderators could be explored to reveal the possible influences on the formative assessment efficacy, such as the gender, treatment type, teacher factor, etc. The possible influence of different English learning contexts, that is, learning English as a foreign language, a second language or a native language, on the formative assessment could be an area for exploration as well.

\section{Funding}

This paper is part of a CSSF (China Social Science Fund) supported project (No: 17BYY100), which is entitled as "The recontextualisation and realisation of formative assessment in the EFL area of China". 


\section{References}

Allal, L. and Lopez, L. (2005). Formative assessment of learning: A review of publications in French Formative Assessment (Eds.). Improving Learning in Secondary Classrooms (pp. 241-264). Paris: OECD Publication.

Bennett, R. E. (2011). Formative assessment: a critical review. Assessment in Education: Principles, Policy \& Practice, $18(1)$, 5-25.

Berry, R. and Adamson, B. (2011). Assessment Reform in Education. Springer Netherlands.

Black, P. and Wiliam, D. (1998a). Assessment and Classroom Learning. Assessment in Education: Principles, Policy \& Practice, 5(1), 7-74.

Black, P. and Wiliam, D. (1998b). Inside the black box: Raising standards through classroom assessment. Phi Delta Kappan, 80(2), 139-144.

Black, P. and Wiliam, D. (2018). Classroom assessment and pedagogy. Assessment in Education: Principles, Policy \& Practice, 25(6), 551-575.

Borenstein, M., Hedges, L. V., Higgins, J. P. T., and Rothstein, H. R. (2009). Introduction to Meta-Analysis. U.K.: Wiley.

Brown, G., Gebril, A., and Michaelides, M. (2019). Teachers' Conceptions of Assessment: A Global Phenomenon or a Global Localism. Front. Educ., 4, 16.

Carless, D. and Lam, R. (2014). Developing assessment for productive learning in Confucian-influenced settings: Potentials and challenges. In C. Wyatt-Smith, V. Klenowski, \& P. Colbert (Eds.), Designing Assessment for Quality Learning (pp. 167-182). Dordrecht: Springer.

CMoE. (2001). Guidelines from the Ministry of Education for promoting primary school English [jiaoyubu guanyu xiaoxue tuixing yingyu jiaoxuekecheng de zhuyao yijian]. Basic education No. [2001] 2. Retrieved August 13, 2008 from http://www.moe.edu.cn/20010907/3000637.html (in Chinese).

CMoE. (2003). English curriculum standards for general high school students. Beijing, China: People’s Education Publishing House (in Chinese).

CMoE. (2007). College English Curriculum Requirements. Retrieved September 27, 2007, fromhttp://www.moe.edu.cn/ edoas/website18/info34295.htm (in Chinese).

Chen, Q., May, L., Klenowski, V., and Kettle, M. (2014). The enactment of formative assessment in English language classrooms in two Chinese universities: Teacher and student responses. Assessment in Education, 21(3), 271-285.

Chen, Q. (2016). Cultural appropriateness and appropriation of formative assessment in the Chinese context. Journal of Shanxi University (In Chinese), 39(3), 80-90.

Cheng, L. and A. Curtis. (2009). English language assessment and the Chinese learner. New York, NY: Routledge.

Cohen, J. (1988). Statistical power analysis for the behavioral sciences (2nd ed.). Hillsdale, NJ: Erlbaum.

CPC Central Committee and State Council. (2020). The overall plan for deepening thereform of education evaluation in the new era. http://www.gov.cn/zhengce/2020-10/13/ content_5551032.htm.

Darwish, Al, S. and Sadeqi, A. A. (2016). Reasons for College Students to Plagiarize in EFL Writing: Students’ Motivation to Pass. International Education Studies, 9(9), 99-110.

Dunn, K. E. and Mulvenon, S. W. (2009). A Critical Review of Research on Formative Assessments: The Limited Scientific Evidence of the Impact of Formative Assessments in Education. Practical Assessment, Research, and Evaluation, 14(7), 1-11.

Duval, S. and Tweedie, R. (2000). A Nonparametric “Trim and Fill” Method of Accounting for Publication Bias in Meta-Analysis. Journal of the American Statistical Association, 95(449), 89-98.

Flórez, T. and Sammons, P. (2013). A literature review of assessment for learning: Effects and impact. Oxford: University of Oxford, Department of Education and CfBT.

Graham, S., Hebert, M., and Harris, K. R. (2015). Formative assessment and writing: A meta-analysis. The Elementary School Journal, 115(4), 523-547.

Han, M. and Yang, X. (2001). Educational assessment in China: Lessons from history and future prospects. Assessment in Education, 8(1), 5-10.

Hattie, J. and H. Timperley, H. (2007). The power of feedback. Review of Education Research, 77(1), 81-112.

Huang, J. and Luo, S. (2014). Formative Assessment in L2 Classroom in China: The Current Situation, Predication and Future. Indonesian Journal of Applied Linguistics, 3(2), 113-127.

Huang, J., Luo, S., and Lin, D. (2019). A Review of Formative Assessment of Foreign Language Education in China: Review and Suggestions. Foreign Language Testing and Teaching, (03), 1-9+43.

Jin, Y. and Sun, H. (2020). Forty Years of the Theory and Practice of Language Testing in China: Retrospect and Prospect. Foreign 
Languages in China, 17(04), 4-11.

Kachru, B. B. (1996). Special Issue: Distinguished Humanities Lectures II || World Englishes: Agony and Ecstasy. Journal of Aesthetic Education, 30(2), 135-155.

Kennedy, K. J. (2016). Exploring the influence of culture on assessment: The case of teachers' conceptions of assessment in Confucian-heritage cultures. In G. T. L. Brown \& L. R. Harris (Eds.), Handbook of human and social conditions in assessment (pp. 404-419). New York: Routledge.

Kingston, N. and Nash, B. (2011). Formative assessment: A meta-analysis and a call for research. Educational Measurement: Issues and Practice, 30(4), 28-37.

Klute, M., Apthorp, H., Harlacher, J., and Reale, M. (2017). Formative assessment and elementary school student academic achievement: A review of the evidence (REL 2017-259). Washington, DC: Regional Educational Laboratory Central.

Lee, H. C., Huy Q., Zhang, Y., Abedi, J., and Warschauer, M. (2020). The Effectiveness and Features of Formative Assessment in US K-12 Education: A Systematic Review. Applied Measurement in Education, 33, 124-140.

Li, S. and Wang, H. (2018). Traditional literature review and research synthesis. In A. Phakiti, P. De Costa, L. Plonsky, \& S. Starfield (Eds.), The Palgrave Handbook of Applied Linguistics Research Methodologies (pp. 123-144). London: Palgrave Macmillan.

Liu, J. and Xu, Y. (2017). Assessment for Learning in English Language Classrooms in China: Contexts, Problems, and Solutions. In H. Reinders, D. Nunan \& B. Zou (Eds.), Innovation in Language Learning and Teaching: The Case of China (pp. 17-37). London: Palgrave Macmillan UK.

Min, S., He, L., and Zhang, J. (2020). Review of recent empirical research (2011-2018) on language assessment in China. Language Teaching, 53(3), 1-25.

Moher, D., Liberati, A., Tetzlaff, J., and Altman, D. G. (2009). Preferred reporting items for systematic reviews and meta-analyses: The PRISMA statement. Annals of Internal Medicine, 151(4), 264-269.

Poole, A. (2016). 'Complex teaching realities' and 'deep rooted cultural traditions': Barriers to the implementation and internalisation of formative assessment in China. Cogent Education, 3(1):1-14.

Qi, L. (2007). Is testing an efficient agent for pedagogical change? Examining the intended washback of the writing task in a high-stakes English test in China. Assessment in Education: Principles, Policy \& Practice, 14(1), 51-74.

Rea-Dickins, P. (2004). Understanding teachers as agents of assessment. Language Testing, 21(3), 249-258.

Shute, V. (2007). Focus on formative feedback (ETS Research Report RR-07-11). Princeton, NJ: Educational Testing Service.

U.S. Department of Education. (2001). No Child Left Behind fact sheet. Washington, DC: U.S. Government Printing Office.

$\mathrm{Xu}, \mathrm{Y}$. and Harfitt, G. (2018). Is assessment for learning feasible in large classes? Challenges and coping strategies from three case studies. Asia-Pacific Journal of Teacher Education, 47, 1-15.

Yin, Y. (2005). The influence of formative assessments on student motivation, achievement, and conceptual change. Unpublished doctoral dissertation. Harvard University, Cambridge, MA.

Yuan, S. and Shu, D. (2017). Formative assessment studies in foreign language teaching in China: Review and reflection (2002-2016). Foreign Language Learning Theory and Practice, (4), 51-56. 